\title{
Functional genomic analysis of glioblastoma multiforme through short interfering RNA screening: a paradigm for therapeutic development
}

\author{
Nikhil G. Thaker, B.S., ${ }^{1}$ Fang Zhang, Ph.D., ${ }^{2-4}$ Peter R. McDonald, Ph.D., ${ }^{2-4}$ \\ Tong Ying Shun, Ph.D., ${ }^{2-4}$ John S. Lazo, Ph.D., ${ }^{2-4}$ and Ian F. Pollack, M.D. ${ }^{5}$ \\ ${ }^{1}$ University of Medicine and Dentistry of New Jersey-New Jersey Medical School, Newark, New Jersey; \\ Departments of ${ }^{2}$ Pharmacology and ${ }^{3}$ Chemical Biology, ${ }^{4}$ Drug Discovery Institute, University of Pittsburgh, \\ Pittsburgh, Pennsylvania; and ${ }^{5}$ Department of Neurological Surgery, University of Pittsburgh, Pittsburgh, \\ Pennsylvania
}

\begin{abstract}
Glioblastoma multiforme (GBM) is a high-grade brain malignancy arising from astrocytes. Despite aggressive surgical approaches, optimized radiation therapy regimens, and the application of cytotoxic chemotherapies, the median survival of patients with GBM from time of diagnosis remains less than 15 months, having changed little in decades. Approaches that target genes and biological pathways responsible for tumorigenesis or potentiate the activity of current therapeutic modalities could improve treatment efficacy. In this regard, several genomic and proteomic strategies promise to impact significantly on the drug discovery process. High-throughput genome-wide screening with short interfering RNA (siRNA) is one strategy for systematically exploring possible therapeutically relevant targets in GBM. Statistical methods and protein-protein interaction network databases can also be applied to the screening data to explore the genes and pathways that underlie the pathological basis and development of GBM. In this study, we highlight several genome-wide siRNA screens and implement these experimental concepts in the T98G GBM cell line to uncover the genes and pathways that regulate GBM cell death and survival. These studies will ultimately influence the development of a new avenue of neurosurgical therapy by placing the drug discovery process in the context of the entire biological system. (DOI: 10.3171/2009.10.FOCUSO9210)
\end{abstract}

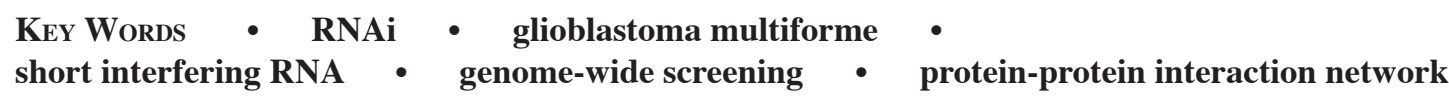

$\mathrm{G}$ LIOBLASTOMA multiforme is the most common form of primary human brain tumor,,$^{30}$ and despite major advances in the management and treatment of these tumors, the prognosis remains dismal. Resistance of GBM to conventional chemotherapy and radiation therapy has necessitated a search for more effective therapies, which are beginning to encompass modern molecular biology and drug discovery techniques to identify and target the specific molecular genetic aberrations that underlie the pathogenesis of GBM. ${ }^{58}$ However, the results of first-generation clinical trials with molecularly targeted agents have generally been disappointing, owing to tumor heterogeneity and an incomplete understanding of the interconnecting molecular pathways that promote and maintain tumor growth.

Therapeutic strategies that target genes and biologi-

\footnotetext{
Abbreviations used in this paper: GBM = glioblastoma multiforme; IPA = Ingenuity Pathways Analysis; KEGG = Kyoto Encyclopedia of Genes and Genomes; MAD = median of the absolute deviation; RISC = RNA-induced silencing complex; RNAi = RNA interference; siRNA = short interfering RNA; TNF $=$ tumor necrosis factor; V-ATPase $=$ vacuolar type $\mathrm{H}^{+}$-ATPase .
}

cal pathways responsible for the development of tumors or potentiation of current therapies could improve patient outcomes. Accordingly, several genomic and proteomic methodologies promise to expand the current drug discovery process. High-throughput screening with siRNA is one strategy for systematically exploring the possible therapeutically relevant targets in cancers, such as GBM. Short interfering RNA are 20-25 nucleotide-long double-stranded RNA molecules that can selectively silence specific genes through sequence-specific mRNA transcript degradation..$^{55,57}$ The availability of siRNA libraries and automated liquid handling platforms have spawned an evolution in genome-wide investigations of loss-offunction phenotypes. ${ }^{25,30,52,75,77}$ However, this genomics approach has not yet been implemented in neurooncology and will require an analysis of biological pathways as a central reference point to provide a global perspective on the development, function, and pathological basis of neurosurgical disease.

In this study, we describe the drug discovery process, considerations and applications of genome-wide siRNA screening, and the integration of high-content statistical 

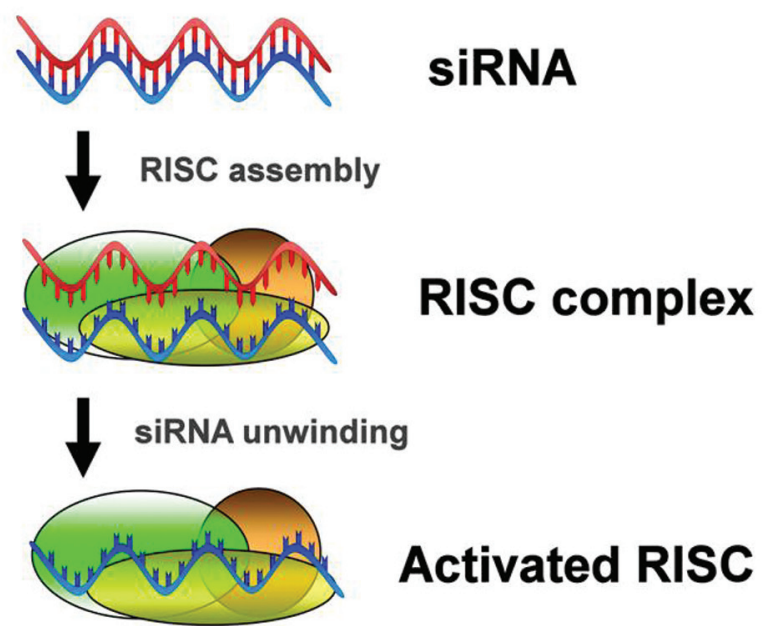

$\downarrow$ Target mRNA recognition

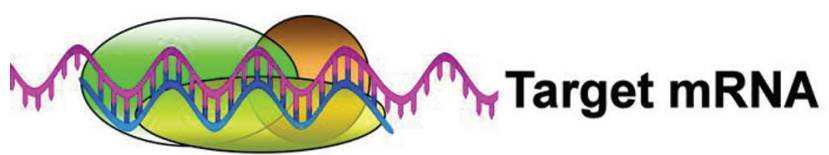

$\checkmark$ mRNA target cleavage

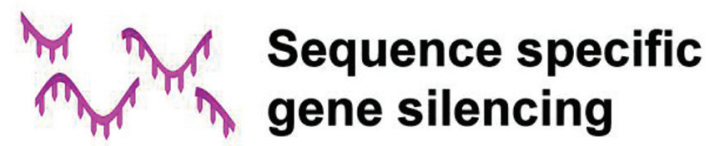

mRNA degradation

FIG. 1. The mechanism of RNAi. Long double-stranded RNA molecules are cleaved by the RNase-III-like enzyme Dicer into siRNA molecules 20-25 base pairs long with 3' base pair overhangs. Synthesized siRNA molecules may be directly transfected into cells and do not undergo processing with Dicer. The antisense strand of this siRNA molecule is then incorporated in the RISC complex. This sequence binds to a complementary sequence on an mRNA, and an RNase within the RISC complex cleaves and destroys the mRNA by endonucleolytic cleavage, resulting in silencing of gene expression and reduction of protein levels (Kittler and Buchholz; Martinez et al.).

methods and protein-protein interaction network databases. We then highlight several high-throughput genomewide siRNA screens in a spectrum of disease models and use these experimental concepts to implement a highthroughput siRNA screen in the T98G GBM cell line to uncover the genes and pathways that modulate GBM cell death and survival.

\section{Drug Discovery and the Druggable Genome}

The identification and validation of novel drug targets has remained difficult and costly, despite advances in molecular biology, molecularly targeted therapies, and highthroughput screening technologies. Techniques that target gene expression can facilitate the drug discovery process by replicating the potential effects of pharmacologically blocking a given protein, thereby providing insights for the drug development process. In recent years, siRNA has become a powerful tool for assessing the loss-of-function

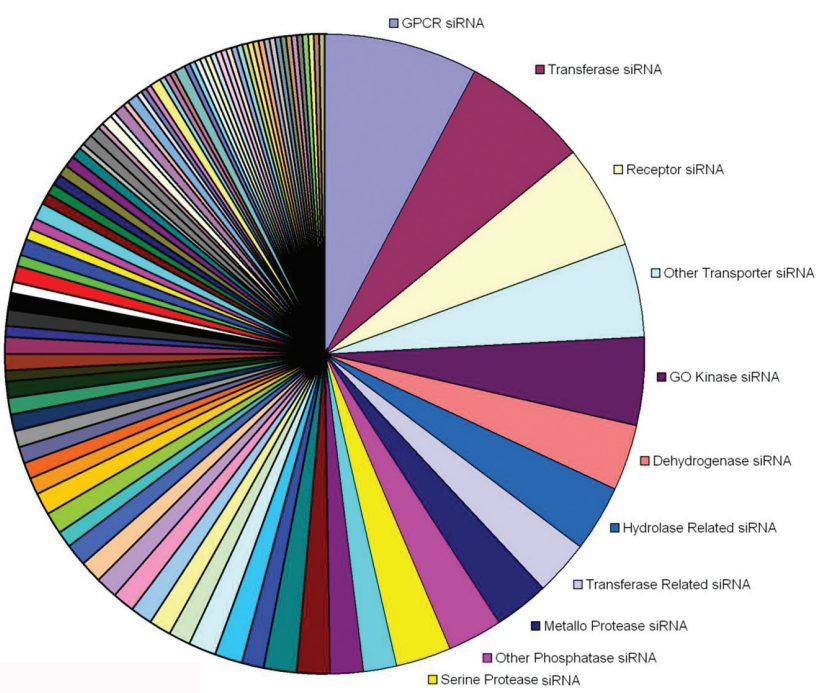

Fig. 2. Commonly targeted gene families of the druggable genome. These gene families represent the genes that are targeted by RNAi screening libraries. This pie chart is approximately based on the Ambion Silencer Druggable Genome siRNA Library version 1.0. GO = gene ontology; GPCR = G-protein coupled receptor.

phenotype associated with protein knockdown within the cell. In the RNAi mechanism, gene expression is silenced through sequence-specific mRNA transcript degradation modulated by sequence complementarity within the RNA-induced silencing complex (RISC) ${ }^{4}$ (Fig. 1). Short interfering RNA technology has also facilitated multiple steps of the drug discovery process, which includes target identification, target validation, compound screening, lead optimization, and clinical applications.

\section{Druggable Genome}

The drug discovery process may be enhanced through an assessment of the genes and proteins that represent opportunities for therapeutic intervention. ${ }^{24}$ Whole-genome sequencing has facilitated the functional annotation of a list of prospective drug targets, and "in silico" experimental molecular techniques have further allowed refinement of the list of molecules that can be targeted with drugs or drug-like molecules. ${ }^{24,56}$ Analysis of this so-called "druggable genome" provides a basis for tailoring drug discovery efforts to focus on a high-yield subset of genes, and RNAi libraries have been developed to consist of molecules that specifically target these druggable proteins ${ }^{48}$ (Fig. 2). Given the high attrition rate with conventional drug development strategies, this approach promises to rapidly assess and prioritize the most therapeutically promising targets.

\section{Implementing siRNA Screens}

Genome-wide screening has significantly contributed to our understanding of biology; these studies have examined signaling pathways, disease-associated genes, and genes involved in viability, secretion, chromosome segregation, neuron development, and neuron outgrowth. ${ }^{11,18-20,26,36,38,40,42,45,60,65,67,69,70,74,75}$ Short interfering 


\section{Functional genomic analysis of siRNA screens}

TABLE 1: Summary of genome-wide RNAi screens in mammalian model systems

\begin{tabular}{|c|c|c|}
\hline Authors \& Year & Model & Summary \\
\hline Whitehurst et al., 2007 & non-small cell lung cancer & paclitaxel chemosensitivity \\
\hline Giroux et al., 2006 & pancreatic adenocarcinoma & spontaneous apoptosis \& gemcitabine chemosensitivity \\
\hline Tu et al., 2009 & adipocytes & insulin signaling pathway constituents that modulate insulin resistance \\
\hline Ganesan et al., 2008 & melanocytes & genes \& pathways that modulate melanogenesis \\
\hline Leal et al., 2008 & mouse embryonic fibroblast & downregulation of S-adenosylhomocysteine contributes to tumorigenesis \\
\hline Tang et al., 2008 & cervical \& colorectal cancer & TCF transcription factors identified in Wnt pathway activation \\
\hline Sepp et al., 2008 & embryonic cerebral cortical neurons & identifying neural outgrowth genes \\
\hline Loh et al., 2007 & neuroblastoma & kinase cluster required for neurite outgrowth \& retraction \\
\hline Hu et al, 2009 & mouse embryonic stem cells & transcriptional modules required for self-renewal \\
\hline Westbrook et al., 2005 & $\begin{array}{l}\text { human mammary epithelial cells \& } \\
\text { colorectal cancer }\end{array}$ & identification of a previously unrecognized tumor suppressor REST \\
\hline Luo et al., 2009 & Ras mutant cells & $\begin{array}{l}\text { identification of PLK1 \& the proteasome as synthetic lethal targets in Ras } \\
\text { mutant cells }\end{array}$ \\
\hline
\end{tabular}

RNA screening offers an unbiased, systematic strategy for uncovering the biological genes or pathways that underlie disease processes, allowing a deeper understanding of the poorly described molecular mechanisms governing various cellular processes or diseases.

The essential first step in the cancer drug discovery process is the identification of novel drug targets. Recent target identification has relied on characterization of genomic mutational spectra or proteomic expression signatures for correlation of target identification or tumor response to therapies. ${ }^{1,50}$ In this context, siRNA screening represents a complementary hypothesis-generating approach by defining the consequences of blocking a given target. The events that underlie tumorigenesis, tumor progression, and tumor response to conventional therapies each represent excellent targets for selective killing of cancer cells versus normal cells that may be delineated on an siRNA-based screen, although the potential applications of these approaches in neurooncology and neurosurgery may have relevance from both a therapeutic and mechanistic perspective. ${ }^{38,60}$ Recent siRNA screens in cancer and disease models have focused on: 1) reversing the cancer phenotype, 2) identifying synthetic lethal targets, 3) developing synergistic drug combinations, and 4) clarifying underlying mechanisms of biological processes (Table 1).

\section{Reversing the Cancer Phenotype}

The cancer phenotype is associated with several welldefined hallmarks, including self-sufficiency in growth signals, insensitivity to antigrowth signals, evasion of apoptosis, limitless replicative potential, sustained angiogenesis, tissue invasion and metastasis, and genome instability. ${ }^{22,30}$ Notably, invasion and metastatic spread of cancer are complex biological processes that are directly involved in the pathological process. ${ }^{11}$ In an siRNA screen targeting 5234 human genes in an ovarian carcinoma cell line, the authors identified the potential therapeutic utility of targeting mitogen-activated protein kinase pathway in cancer progression. ${ }^{11}$ This effect was also reproduced with a small-molecule inhibitor of c-Jun $\mathrm{N}$-terminal kinase (JNK). In another genome-wide RNAi study, the authors described the role of Growth arrest-specific 1 as a novel tumor suppressor gene that effectively suppressed melanoma metastasis. ${ }^{20}$ Furthermore, another RNAi screen identified a novel tumor suppressor gene REST/NRSF, which is a transcriptional repressor of neuronal gene expression, in human mammary epithelial cells and observed its associated frequent deletion in colorectal cancer cells. ${ }^{74}$ These studies demonstrate the ability of RNAi screening to identify novel targets that may represent cruxes of the cancer phenotype, and the identified proteins may represent nodes of chemosensitivity in various disease models.

\section{Synthetic Lethality}

Of utmost importance to clinicians is the development of novel combination therapies that can be swiftly translated into clinical application. Modern drug discovery aims to create novel drug combinations that will selectively kill cancer cells while leaving normal cells unharmed. However, this process has been difficult, owing to exploitation of normal enzyme functionality by oncogenes and the inability to pharmacologically target tumor-suppressor genes that have low or absent activity. ${ }^{28}$ Synthetic lethality holds promise to evade some of these difficulties, and RNAi screening can be used to identify these synthetic lethal relationships using high-throughput technologies. Two genes are considered synthetic lethal if mutation of either is compatible with cell viability but 
N. G. Thaker et al.

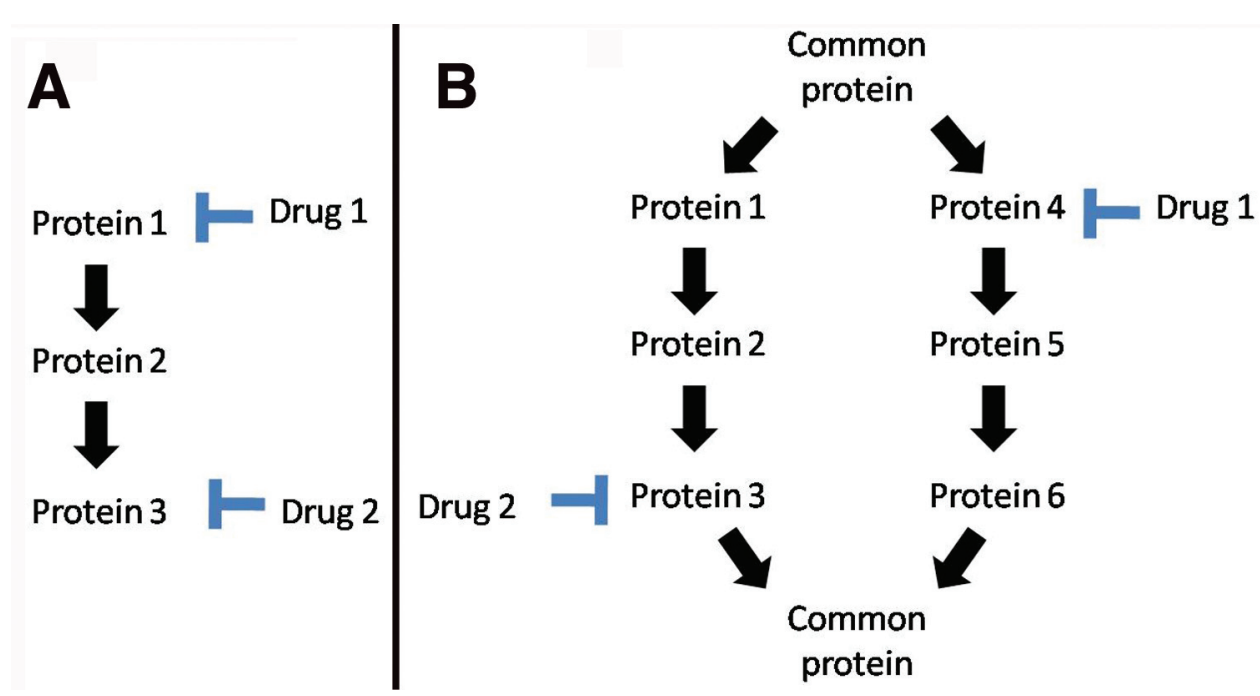

FIG. 3. Schematic diagram depicting abrogation of protein function in single, parallel, converging, and diverging pathways. A: Abrogation of enzymatic or protein function in a single, essential pathway can occur upstream or downstream in the pathway. B: Abrogation of 2 proteins simultaneously in a parallel pathway, a converging pathway, or a diverging pathway can be assessed with synthetic lethal or chemosensitizer screening assays.

mutation of both leads to cell death. ${ }^{31}$ Once this screening tool has identified such genes, anticancer therapies can be developed to target the molecular pathways. For instance, mutation of 2 essential components on 1 linear pathway, such as Proteins 1 and 3 in Fig. 3A, or mutation of 2 components of parallel, converging, or diverging pathways, such as Proteins 3 and 4 in Fig. 3B, may be synthetically lethal.

A well-known synthetic lethal relationship has been demonstrated by the inhibition of poly (ADP-ribose)polymerase-1 (PARP1) in breast cancer cells deficient in $B R C A 1, B R C A 2$, or other components of the homologous recombination pathway, while normal cells remain unaffected. ${ }^{8,15,16,59,70}$ In normal cells, both the homologous recombination and base-excision repair pathways repair damaged DNA; PARP1 is an enzyme required for baseexcision repair, which is a pathway that repairs singlestrand breaks; $B R C A 1$ and $B R C A 2$, which are tumor-suppressor genes, are required for DNA double-strand break repair by homologous recombination, and mutations in $B R C A 1$ and $B R C A 2$ predispose to breast and ovarian carcinomas. Loss of PARP1 increases DNA damage repair through the homologous recombination pathway; thus, abrogation of the homologous recombination pathway concomitant with base-excision repair pathway inhibition could lead to significant cell death. Indeed, recent studies have shown that BRCAI or BRCA2 mutation or absence sensitizes cells to inhibition of the PARP1 enzyme, ${ }^{15}$ and patients with hereditary breast or ovarian cancers may be excellent candidates for treatment with PARP1 inhibitors.

This synthetically lethal relationship was deduced by leveraging current understanding of cell biology and the known molecular genetic alterations within cancer. However, additional synthetically lethal combinations may not be readily deduced through a rational mechanistic understanding of cancer cell biology, and an unbiased screening method is needed to systematically detect novel rela- tionships. In a recent genome-wide RNAi study, Turner et al..$^{70}$ identified targets that modulated the sensitivity of breast cancer cells to the effects of PARP1 inhibition. Silencing of several kinases strongly sensitized PARP1 inhibition, and these targets included cyclin-dependent kinase 5 (CDK5), MAPK12, PLK3, PNKP, STK22c, and STK36. The presence of CDK5, which is required for DNA-damage checkpoint activation, suggests that normal checkpoint function may be essential for DNA repair when the PARP1 enzyme is inhibited. ${ }^{28,70}$ Genome-wide RNAi screens, therefore, offer a unique opportunity to implement a systematic, rapid, and unbiased method to uncover novel synthetic lethal relationships.

\section{Synergistic Drug Combinations}

Despite promising in vitro and in vivo data, intrinsic or acquired resistance to conventional therapies has been a major therapeutic obstacle. Tumor heterogeneity, redundancy and parallel processing of intracellular signaling pathways, inactivating metabolism, mutation within a specific targeted pathway, loss of negative inhibition, mutations leading to constitutive activation, and limited drug delivery are the most common resistance mechanisms. ${ }^{51}$ Given these therapeutic challenges and the multiple mutations leading to tumorigenesis, tumor cells will need to be targeted with several agents simultaneously to ensure a cure or long-term survival. Combination therapies that target multiple signaling pathways or different constituents in the same pathway (Fig. 3) may overcome resistance mechanisms and widen the therapeutic window, ultimately enhancing the effect on tumor cells without increasing toxicity for normal cells. However, therapeutic combinations are limitless, and a strategy is necessary to select only the most effective and synergistic of combinations.

Investigators have used RNAi screens to identify targets that chemosensitize cancer cells to conventional treatments or molecularly targeted therapies ${ }^{19,42,45,75}$ (Table 


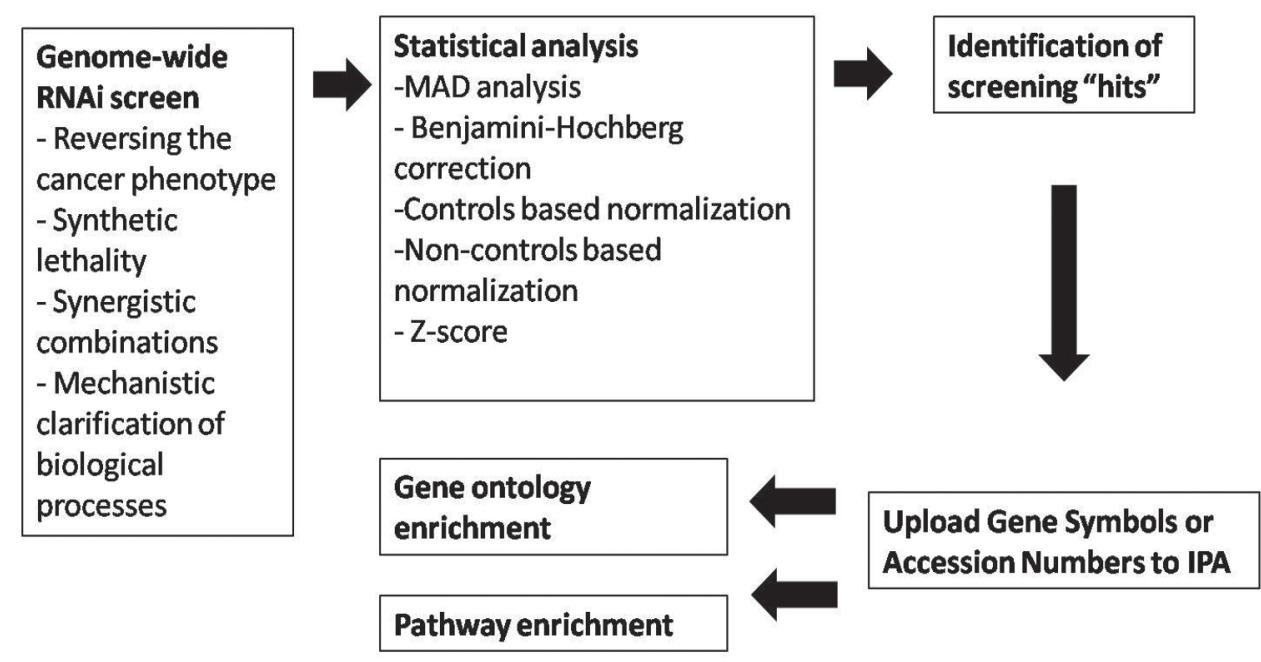

FIG. 4. Functional genomic analysis through genome-wide siRNA screening. This flow diagram depicts the chronology of events in the implementation and functional genomic analysis of a genome-wide siRNA screen. After statistical analysis with one or several methods, screening "hits" are uploaded to the web-based protein-protein interaction network IPA. Gene ontology enrichment is conducted with the Fisher exact test at $\alpha=0.05$ by comparing the "hits" to a list of ontology categories. Pathway enrichment is similarly conducted by comparing "hits" to the KEGG list of pathways.

1). In the most well-defined of these studies, Whitehurst et al. ${ }^{75}$ identified gene loci that chemosensitized nonsmall cell lung cancer cells to the microtubule stabilizer paclitaxel. As a proof of principle, the authors identified several proteasome components as chemosensitizing targets, and proteasome inhibitors have been shown to enhance paclitaxel-induced apoptosis in several cancers. ${ }^{41,49}$ The authors also reported that exposure to the V-ATPase inhibitor salicylihalamide A combined with low concentrations of paclitaxel achieved a synergistic decrease in cell viability. Such proof-of-principle synergism studies are also being applied to neurooncology models, which will be described below.

\section{Mechanistic Clarification of Biological Processes}

Systematic phenotyping by means of RNAi and other approaches will also provide novel perspectives on a gene's or pathway's function in the context of the genome. Function and development of the brain require the coordinated action of numerous genes, but we currently understand the functions of only a small fraction of them. ${ }^{60}$ The integration of phenotypic information from genomic and proteomic data sets has revealed many important cellular processes at an unprecedented resolution. ${ }^{17}$ There are several such studies in neuroscience that have provided insight into the development and function of the nervous system, including neural outgrowth ${ }^{60}$ and identification of kinase clusters that are required for neurite outgrowth and retraction ${ }^{38}$ (Table 1).

Utilizing an RNAi screening approach, a recent study identified a pathogenic link between the endocytic pathway and neuronal dysfunction in synucleinopathies, such as Parkinson disease. ${ }^{34}$ Other RNAi studies have focused on dissecting various cell processes, such as insulin signaling, melanogenesis, Wnt pathway signaling, and self-renewal (Table 1), and this screening strategy holds promise for the mechanistic clarification behind brain development, function, and the pathological basis of diseases, such as GBM.

\section{Analyzing RNAi Screens}

With the large amount of data generated from siRNA screening, there is a clear need for data reduction methods, which would allow prioritization of targets and determination of the gene expression products most significantly affected by siRNAs in the disease model. ${ }^{9}$ Screening typically relies on sophisticated automation, appropriate controls, and state-of-the-art detection technologies to organize and analyze thousands of test samples. ${ }^{5,7}$ Unfortunately, siRNA screening is associated with "off-target" effects, which can affect the analysis and final results. Data output, therefore, requires sophisticated and rigorous analysis methods to reduce the number of high-confidence "hits." Statistical analyses and the use of proteinprotein interaction network databases are 2 such methods that can facilitate the data analysis process. An overview of several such data analysis methodologies is depicted in Fig. 4.

\section{Statistical Analysis}

Although a comprehensive review of statistical methods for high-throughput screening "hit" selection is beyond the scope of this review, we present the most popular methods with accompanying references for further review. As with any high-throughput methodology, the output varies due to: 1) systematic variation, or 2) unsystematic, random influences. Systematic effects that are not adjusted for can bias the final results of the screen, creating false-positive and false-negative results. The level of random "noise" can also similarly confound the results of a study and therefore also needs to be accounted for in the "hit" selection process.

Most high-throughput screens are conducted in 384- 
well plate formats, and most well-designed experiments will include an in-plate positive control (a control that will yield a positive result; for example, cell death in a toxicity assay) and a negative control (a control that will yield a negative result; for example, minimal cell death in a toxicity assay). These controls are used to normalize the wells with targeting siRNAs. Although this methodology is frequently used and is the traditional way that biologists view changes in biological activity, controls-based methods have several potential problems. These include positional variability based on the location of the well on the plate; systematic biases among the controls; variability between control wells; and outliers due to measurement problems. ${ }^{7,12}$

With these issues in mind, some investigators are utilizing non-controls-based normalization, such as normalizing to the median of all values on a plate. The median, unlike the mean, is not affected by outliers. Because it is the outliers on a plate that may be of greatest interest (that is, those wells that had the greatest amount of cancer cell death), this method would help distinguish outliers from the majority of the screening plate. Several popular analysis methods include Z-scores ${ }^{76}$ viability ratios and Benjamini-Hochberg correction, ${ }^{75}$ median of the absolute deviation (MAD) method,,$^{10,23,29}$ other non-controls-based normalization methods, ${ }^{7,12}$ and orthogonal analysis methods based on a combination of these statistical methods. ${ }^{75}$ We have uniquely developed and applied the MAD method for the detection and removal of outlier data points from high-throughput screening data and further describe our techniques below.

\section{Protein-Protein Interaction Network Analysis}

Despite many decades of experiments and many thousands of data points, the cellular and molecular functions of the cancer genome or proteome have not yet been systematized. Because of the complexity of the cellular network, biologists have preferred to consider parts of it by subdividing it into biological pathways that comprise sets of molecules involved in a particular function or process. ${ }^{2}$ Pathways can therefore serve as a scaffold for assessing the impact of single molecules on the network of cellular proteins. For instance, proteins that connect to numerous molecules within interaction networks are more likely to produce a cytotoxic effect when deleted (knocked down), whereas proteins that may be part of parallel or redundant biological pathways are less likely to cause lethality when deleted (Fig. 3). Several commercially available proteinprotein interaction network databases ${ }^{13,14,68}$ have focused on the pathways relevant to human disease, and these interaction analyses can be used in the target identification and validation phases of the drug discovery process.

Protein-protein interaction network analysis can uncover the underlying enriched (over-represented) gene functions and pathways that may not be readily apparent otherwise. Subsequent gene ontology and pathway enrichment analyses are then used to narrow down the list of "hits" to uncover biological functions that are most significantly affected by siRNAs ${ }^{21}$ (Fig. 4). To allow functional enrichment according to existing functional annotation systems, the Fisher exact test is adopted to mea- sure gene enrichment of annotation classifications such as Gene Ontology terms $\mathrm{s}^{21,63}$ or pathways from the KEGG database. ${ }^{32}$ For each annotation term, the Fisher exact probability describes the probability of sampling without replacement from a finite population consisting of 2 types of elements, and an analogous approach is implemented for the pathway enrichment of siRNA targets. Such an analysis can now be performed using open-access interaction network analyses. ${ }^{27}$

Several studies have implemented this analysis on gene expression data and RNAi screening data ${ }^{6,46,69,72}$ For instance, Bredel et al. ${ }^{6}$ identified 3 novel MYC-interacting genes in human gliomas through functional network analysis of gene expression data with Ingenuity Pathways Analysis (IPA); Mori et al ${ }^{46}$ reported that gene profiling and pathway analysis helped to elucidate the molecular mechanisms involved in neuroendocrine transdifferentiation of prostate cancer cells; and Tu et al. ${ }^{69}$ used an siRNA screening approach to identify a reliable set of components/modulators of the insulin signaling pathway. Thus, functional genomic analyses that leverage multiple types of information have begun to show promise in uncovering important biology not apparent from standard analysis methods.

\section{Druggable Genome-Wide siRNA Screening in GBM: Working Examples}

Cancer cell survival depends on the balance of signaling through survival and apoptotic pathways. ${ }^{66} \mathrm{An}$ increase in survival signaling, through increased survival factors or decreased apoptotic signaling, could confer a proliferative advantage, which may ultimately enhance chemoresistance. Conversely, uncovering the genes or pathways that are most essential for cancer cell survival may enhance the drug discovery process by identifying promising drug targets. To identify the genes and pathways that represent these chemoresistance and chemosensitivity nodes in GBM, we conducted several druggable genome-wide siRNA screens to identify the gene nodes and pathways that modulate GBM cell death and survival.

\section{Methods}

We used a high-throughput siRNA screen with 16,560 siRNAs targeting 5520 unique human genes in the T98G GBM cell line. We selected the T98G cell line because it is a widely available, GBM-derived, human cell line, with a well-characterized radioresistance and chemoresistance profile, ${ }^{61,62,73}$ and it provides an in vitro surrogate for the identification and subsequent validation of novel therapeutic drug targets for GBM. We measured cell viability at 96 hours after siRNA transfection with a resazurin fluorescent dye assay and normalized the targeting siRNA wells to in-plate positive and negative controls. Viability ratios were calculated by normalizing cell viabilities to the overall median cell viability of all 5520 genes when averaged over the screening replicates.

For the siRNA-only screen, we selected the viability ratios that were 3 SDs above the median after statistical analysis with the MAD method (see Statistical Analysis 


\section{Functional genomic analysis of siRNA screens}

TABLE 2: Protective genes in the T98G GBM cell line

\begin{tabular}{|c|c|c|c|}
\hline Gene Symbol & Gene Accession & Full Gene Name & $\begin{array}{c}\text { Viability } \\
\text { Ratio }\end{array}$ \\
\hline TRPC4AP & NM_199368 & transient receptor potential cation channel, subfamily $\mathrm{C}$, member 4 associated protein & 1.544873 \\
\hline SSTR2 & NM_001050 & somatostatin receptor 2 & 1.514676 \\
\hline RHOV & NM_133639 & ras homolog gene family, member $\mathrm{V}$ & 1.514118 \\
\hline FLJ10858 & NM_018248 & nei endonuclease VIII-like 3 (E. coli) & 1.497508 \\
\hline NR2F2 & NM_021005 & nuclear receptor subfamily 2 , group F, member 2 & 1.493387 \\
\hline TAS2R39 & NM_176881 & taste receptor, type 2, member 39 & 1.49172 \\
\hline NR4A2 & NM_173172 & nuclear receptor subfamily 4, group A, member 2 & 1.491645 \\
\hline PGAM1 & NM_002629 & phosphoglycerate mutase 1 (brain) & 1.480993 \\
\hline RHOBTB3 & NM_014899 & Rho-related BTB domain containing 3 & 1.477528 \\
\hline GNAL & NM_182978 & guanine nucleotide binding protein (G protein), alpha activating activity polypeptide, olfactory type & 1.469045 \\
\hline
\end{tabular}

above) and classified this set of genes by shared molecular and biological functions using the protein analysis through evolutionary relationships classification system (PANTHER). ${ }^{44}$ We then uploaded these lists of "hits" to a web-based application for analysis of biological functions, disease categories, toxicological categories, canonical signaling pathways, drug inhibitors, and pathway and gene ontology enrichment analysis. ${ }^{68}$ The Fisher exact test was used with $\alpha=0.05$ to calculate the probability that each function and pathway classification assigned to the set of survival genes was due to chance. This procedure is detailed in Fig. 4.

\section{Results and Discussion}

We identified 16 targeting siRNA reactions that resulted in a significant increase in cell viability (Table 2). Knockdown of these genes appear to enhance T98G GBM cell survival, suggesting that these genes may be functionally associated with cell death pathways. Interestingly, the products of these genes function as nuclear hormone receptors, GTPases, G-protein coupled receptors, oxidases, and mutases, while several genes were unclassified. Utilizing a knowledge-based interaction network, we found that these genes have been implicated in various biological processes including neurological disease, genetic disease, cellular movement, nervous system development and function, and cell signaling. Nine of 16 genes were reportedly overexpressed in primary or secondary glioma, ${ }^{54}$ which provided further clinical evidence of the importance of these nodes in glioma cells. The most statistically significant protein-protein interaction network consisted of genes implicated in gene expression, cell death, and endocrine system disorders and was centered around beta-estradiol, TNF, and the NF- $\mathrm{KB}$ complex (Fig. 5). Recent studies have described the role of the NF- $\kappa \mathrm{B}$ pathway in resistance to TNF-mediated cell death in human glioma, and its role in inflammation, tumor growth, immunity, and an invasive phenotype. ${ }^{53,64}$ Thus, our results highlight several cellular factors and complexes that may be implicated in GBM cell death pathways.

In our recent work, we implemented an siRNA screen to uncover the core genes and pathways that are essential for GBM cell survival (that is, where siRNAinduced protein knockdown induced cell death). ${ }^{68}$ Interestingly, several identified genes were components of the proteasome complex, suggesting that these components may be essential for cell survival or proteasome structure or function or may have the most rapid protein turnover. Further mechanistic validation has shown that disruption of these components may actually induce instability of the proteasome complex by accumulating intermediate forms, which could contribute to loss of cell viability. Furthermore, using the protein-protein interaction network database, we identified clusters of cellular processes that included protein ubiquitination, purine and pyrimidine metabolism, nucleotide excision repair, and NF- $\mathrm{KB}$ signaling, among others. Overall, these findings regarding the significance of the proteasome complex in GBM cell survival represent an unpredicted observation that would not have been obtained without an siRNA-based screening approach.

However, because GBM is a notoriously heterogenous tumor (with respect to cells from different anatomical regions of a patient's tumor as well as between patients), identifying targets in a single cell line, such as the T98G GBM cell line, may not represent drug targets that are effective and consistent outside of this cell line. While our preliminary investigation with this single cell line is proof of principle for this screening approach, we 
N. G. Thaker et al.

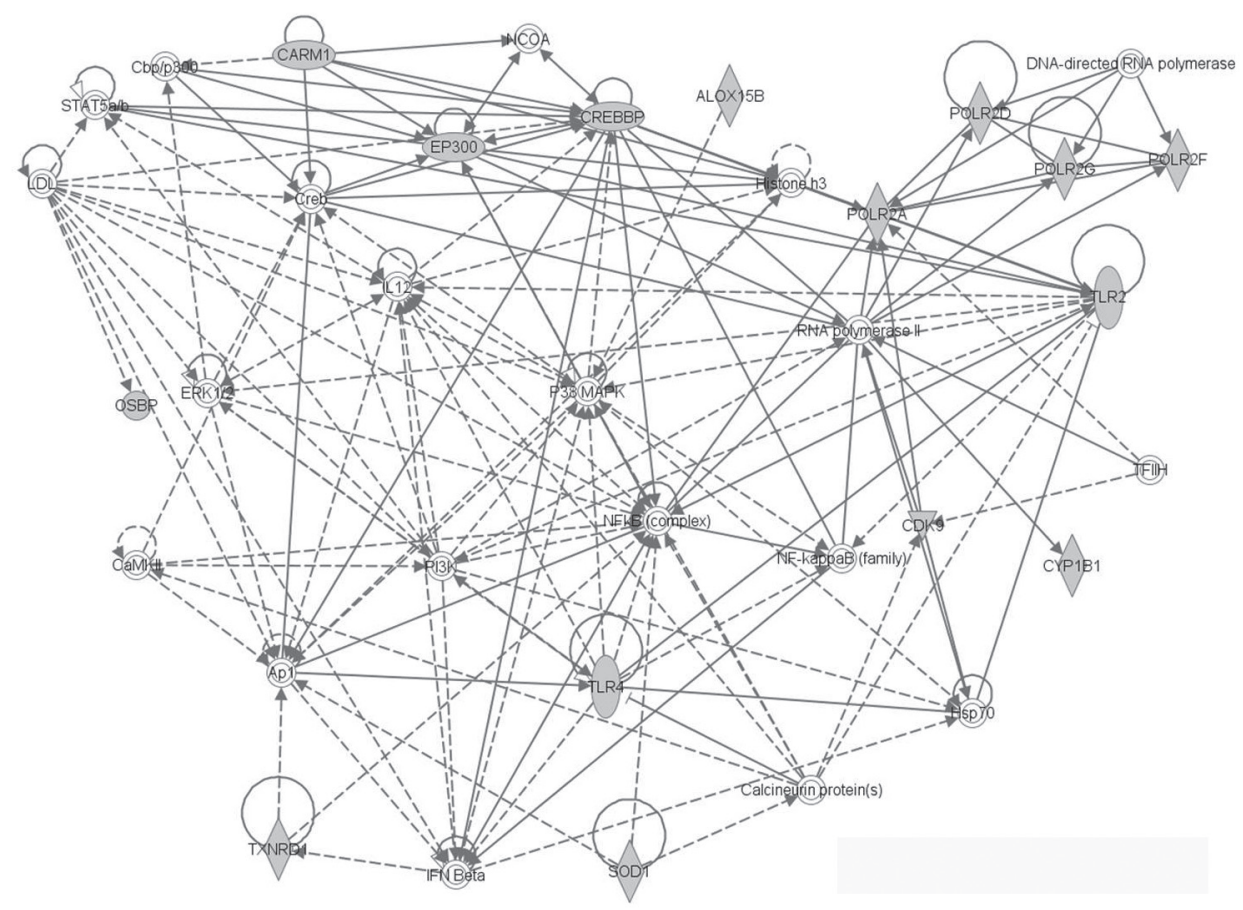

FIG. 5. Mapping of protective genes onto a protein-protein interaction network. Functional analysis of protective genes was performed with IPA. The genes are represented as nodes, and edges connecting 2 nodes represent a biological relationship that is supported by at least 1 published reference or the IPA knowledge base. Shaded nodes represent protective genes. This protein-protein interaction network consisted of genes implicated in gene expression, cell death, and endocrine system disorders and was centered around beta-estradiol, TNF, and the NF-кB complex.

additionally applied this genetic tool to a panel of glioma cell lines and nonglioma cancer cell lines to determine if this approach yielded consistent groups of genomic targets within and between cancer cell lines despite the well-characterized molecular heterogeneity. For instance, the glioma cell lines T98G, U373, U87, LN-Z308, LNZ428, and A172; breast adenocarcinoma cell line MCF7; and lung adenocarcinoma epithelial cell line A549 were transfected with PSMB4 siRNA, and cell viability was measured at 96 hours. The A549 and A172 cell lines were most sensitive, while LN-Z308 and LN-Z428 were most resistant to cell death. Growth inhibition for all cell lines was significantly different from that in control cells ( $p$ $<0.05){ }^{68} \mathrm{We}$ also reproduced these cytotoxic effects using the small-molecule proteasome inhibitor MG-132. ${ }^{68}$ Despite the heterogeneity that exists between and within tumors, screening in a single cell line can yield generalizable results, although they must be ultimately corroborated with a focused secondary screen in other cell lines or primary tumor-derived cell lines. Overall, genome-wide screening in a range of cancer cell lines will continue to provide insight into the similarities and differences in the molecular mechanisms that regulate specific cellular processes.

These studies provide examples of the power and utility of systematic and unbiased functional genomic analysis tools for the identification of novel chemotherapeutic treatment strategies for GBM, and targeting these genes and pathways may provide promising avenues for drug development.

\section{Conclusions and Future Directions}

Recent advances in genomics, such as genome-wide sequencing and the discovery of RNAi, have enabled a detailed study of the integrative nature of cellular signaling and protein-protein interactions. Genome-wide siRNA screening can be used to systematically interrogate the loss-of-function phenotypes associated with protein knock-down and can provide insight into previously unknown gene or pathway functions. Until now, this functional genomics approach has not been applied to the study of neurooncological diseases, such as GBM. In this study, we highlighted several genome-wide siRNA screens conducted in various disease models (Table 1) and then implemented a high-throughput screen to test the protective and synergistic effects of the knockdown of 5520 druggable human genes in the T98G GBM cell line. These studies have yielded a global view of the genes that are implicated in GBM cell survival, chemoresistance, and chemosensitization to various chemotherapeutic agents.

Short interfering RNA screening can be used to develop novel avenues of neurosurgical and neurooncological therapies. The aim of proteomics, which can be described as the study of the role of each gene product in its cellular context, in drug discovery is to identify potential novel drug targets and to achieve a comprehensive description of complex molecular mechanisms. ${ }^{3}$ Once we have identified an siRNA molecule that confers a phenotype of interest (for example, when protein knockdown of gene $\mathrm{X}$ results in cell death), we can focus our efforts on 
the development of a small-molecule inhibitor that can phenocopy the effect of the siRNA. Utilizing this new target for lead optimization, we can then streamline the development of novel monotherapies and combination therapies. Small interfering RNA screening can further impact neurosurgical treatment by identifying promising drug targets, uncovering side-effect profiles of novel and old therapies, allowing rapid assessment of promising drug targets, catalyzing swifter movement through the validation phase of the drug discovery process, identifying novel combination therapies through synthetic lethal and synergism screens, and furthering development of a systems biology understanding of the molecular mechanisms underlying neurological diseases and drug action.

Overall, these genetic studies can be directed toward the therapeutic targeting of essentially any cellular process. This screening approach is not only limited to cell viability assays, as have been described in this work, but can also be used to target various facets of tumor biology, such as tumor microenvironment, tumor invasion, factors that enhance growth in hypoxic conditions, and angiogenesis in a wide range of cell types. This screening tool can also be used to determine the effects of multimodal therapies such as chemo- and radiotherapies on cancer survival. As high-throughput screening technology improves, a more complex assay-end point can be used. For instance, instead of measuring cell viability at the end of the assay, we can measure modulation of angiogenesis using cell-culture and animal models, ${ }^{39}$ cell invasion using migration assays, ${ }^{71}$ and microtubule destabilization using high-content confocal microscopy. ${ }^{47}$ Through these studies, this genetic tool will provide further insight into the mechanisms behind cellular processes and gene functions.

On a systems biology level, gene-expression signatures can now be accurately compared, essentially independent of the platform on which they were generated ${ }^{35}$ The future of high-throughput siRNA screening technology will include integration with DNA microarrays, protein-protein interaction data, and tools like the ConnectivityMap ${ }^{35}$ to provide molecular clarification of novel loss-of-function phenotypes in various cell-based systems. It is also possible that high-throughput proteomic profiling could be combined with siRNA and small-molecule experiments to further inform drug development. However, current cellular networks are incomplete, since only well-studied proteins and interactions are represented (that is, typical nuclear or cytoplasmic proteins). As cancer and disease models become more sophisticated and comprehensive, it will also become important to define standards for communicating genomic profiles across diverse experimental systems. The RNAi technology is allowing the rapid development and implementation of genome-wide screens for disease processes and functions ${ }^{37}$ and will influence the development and understanding of new avenues of neurosurgical therapy.

This genome-wide screening strategy will also have multiple impacts on neurooncological treatments within the clinical setting. For instance, functional genomic profiling of a patient's tumor will enable a more individualized and targeted therapeutic design based on the molec- ular genetic aberrations unique to the tumor's genome. As RNAi transfection technology in primary cells improves, this screening approach will be directly conducted in patient-derived tumor cells, which will in turn allow selection of single-agent or multiagent molecularly targeted therapies that target the biological weaknesses specific to the patient's tumor. ${ }^{51}$ Additionally, this screening strategy will be used to identify the most promising in vitro drug combinations for future clinical trials. By implementing an unbiased, systematic interrogation of the druggable genome, this screening approach will identify novel drug combinations that would not have been identified based on current mechanistic knowledge and will thereby create a list of high-confidence drug combinations for future clinical trials. This screening strategy will also facilitate patient stratification in clinical trials based on the functional significance of specific mutations. Based on these rapidly evolving future screening applications, this screening strategy will significantly impact the clinical approach to individualized genome-based therapies.

\section{Disclosure}

Author contributions to the study and manuscript preparation include the following. Conception and design: NG Thaker, PR McDonald, JS Lazo, IF Pollack. Acquisition of data: NG Thaker, F Zhang. Analysis and interpretation of data: NG Thaker, F Zhang, TY Shun, JS Lazo, IF Pollack. Drafting the article: NG Thaker, IF Pollack. Critically revising the article: NG Thaker, F Zhang, JS Lazo, IF Pollack. Reviewed final version of manuscript and approved it for submission: NG Thaker, F Zhang, JS Lazo. Statistical analysis: TY Shun. Administrative/technical/material support: JS Lazo, IF Pollack. Study supervision: JS Lazo, Ian F Pollack.

This work was supported in part by National Institutes of Health Grants P01 NS40923, P01 CA78039, A168021 to Drs. Lazo and Pollack; and by the Doris Duke Charitable Foundation (N. G. Thaker, fellowship).

\section{References}

1. Anonymous: Comprehensive genomic characterization defines human glioblastoma genes and core pathways. Nature 455: 1061-1068, 2008

2. Apic G, Ignjatovic T, Boyer S, Russell RB: Illuminating drug discovery with biological pathways. FEBS Lett 579:18721877, 2005

3. Bachi A, Bonaldi T: Quantitative proteomics as a new piece of the systems biology puzzle. J Proteomics 71:357-367, 2008

4. Bartz S, Jackson AL: How will RNAi facilitate drug development? Sci STKE 295:pe39, 2005

5. Borawski J, Lindeman A, Buxton F, Labow M, Gaither LA: Optimization procedure for small interfering RNA transfection in a 384-well format. J Biomol Screen 12:546-559, 2007

6. Bredel M, Bredel C, Juric D, Harsh GR, Vogel H, Recht LD, et al: Functional network analysis reveals extended gliomagenesis pathway maps and three novel MYC-interacting genes in human gliomas. Cancer Res 65:8679-8689, 2005

7. Brideau C, Gunter B, Pikounis B, Liaw A: Improved statistical methods for hit selection in high-throughput screening. $\mathbf{J}$ Biomol Screen 8:634-647, 2003

8. Bryant HE, Schultz N, Thomas HD, Parker KM, Flower D, Lopez E, et al: Specific killing of BRCA2-deficient tumours with inhibitors of poly(ADP-ribose) polymerase. Nature 434: 913-917, 2005

9. Calvano SE, Xiao W, Richards DR, Felciano RM, Baker HV, 
Cho RJ, et al : A network-based analysis of systemic inflammation in humans. Nature 437:1032-1037, 2005

10. Chung N, Zhang XD, Kreamer A, Locco L, Kuan PF, Bartz S, et al: Median absolute deviation to improve hit selection for genome-scale RNAi screens. J Biomol Screen 13:149-158, 2008

11. Collins CS, Hong J, Sapinoso L, Zhou Y, Liu Z, Micklash K, et al: A small interfering RNA screen for modulators of tumor cell motility identifies MAP4K4 as a promigratory kinase. Proc Natl Acad Sci U S A 103:3775-3780, 2006

12. Coma I, Herranz J, Martin J: Statistics and decision making in high-throughput screening. Methods Mol Biol 565:69-106, 2009

13. Daraselia N, Yuryev A, Egorov S, Mazo I, Ispolatov I: Automatic extraction of gene ontology annotation and its correlation with clusters in protein networks. BMC Bioinformatics 8:243, 2007

14. Dezso Z, Nikolsky Y, Nikolskaya T, Miller J, Cherba D, Webb $\mathrm{C}$, et al: Identifying disease-specific genes based on their topological significance in protein networks. BMC Syst Biol 3:36, 2009

15. Farmer H, McCabe N, Lord CJ, Tutt AN, Johnson DA, Richardson TB, et al: Targeting the DNA repair defect in BRCA mutant cells as a therapeutic strategy. Nature 434:917-921, 2005

16. Fong PC, Boss DS, Yap TA, Tutt A, Wu P, Mergui-Roelvink $\mathrm{M}$, et al: Inhibition of poly(ADP-ribose) polymerase in tumors from BRCA mutation carriers. N Engl J Med 361:123-134, 2009

17. Fuchs F, Boutros M: Cellular phenotyping by RNAi. Brief Funct Genomics Proteomics 5:52-56, 2006

18. Ganesan AK, Ho H, Bodemann B, Petersen S, Aruri J, Koshy S, et al: Genome-wide siRNA-based functional genomics of pigmentation identifies novel genes and pathways that impact melanogenesis in human cells. PLoS Genet 4:e1000298, 2008

19. Giroux V, Iovanna J, Dagorn JC: Probing the human kinome for kinases involved in pancreatic cancer cell survival and gemcitabine resistance. FASEB J 20:1982-1991, 2006

20. Gobeil S, Zhu X, Doillon CJ, Green MR: A genome-wide shRNA screen identifies GAS1 as a novel melanoma metastasis suppressor gene. Genes Dev 22:2932-2940, 2008

21. Gusev Y: Computational methods for analysis of cellular functions and pathways collectively targeted by differentially expressed microRNA. Methods 44:61-72, 2008

22. Hanahan D, Weinberg RA: The hallmarks of cancer. Cell 100:57-70, 2000

23. Hawkins D: Identification of Outliers. London: Chapman and Hall, 1980

24. Hopkins AL, Groom CR: The druggable genome. Nat Rev Drug Discov 1:727-730, 2002

25. Horvath S, Zhang B, Carlson M, Lu KV, Zhu S, Felciano RM, et al: Analysis of oncogenic signaling networks in glioblastoma identifies ASPM as a molecular target. Proc Natl Acad Sci U S A 103:17402-17407, 2006

26. Hu G, Kim J, Xu Q, Leng Y, Orkin SH, Elledge SJ: A genomewide RNAi screen identifies a new transcriptional module required for self-renewal. Genes Dev 23:837-848, 2009

27. Huang DW, Sherman BT, Lempicki RA: Systematic and integrative analysis of large gene lists using DAVID bioinformatics resources. Nat Protoc 4:44-57, 2008

28. Iglehart JD, Silver DP: Synthetic lethality-a new direction in cancer-drug development. N Engl J Med 361:189-191, 2009

29. Iglewicz B, Hoaglin D: How to detect and handle outliers, in ASQC Basic References in Quality Control: Statistical Techniques. Milwaukee, WI: ASQC Quality Press, 1993, Vol 16

30. Iorns E, Lord CJ, Turner N, Ashworth A: Utilizing RNA in- terference to enhance cancer drug discovery. Nat Rev Drug Discov 6:556-568, 2007

31. Kaelin WG Jr: The concept of synthetic lethality in the context of anticancer therapy. Nat Rev Cancer 5:689-698, 2005

32. Kanehisa M: The KEGG database. Novartis Found Symp 247: 91-103, 119-128, 244-252, 2002

33. Kittler R, Buchholz F: Functional genomic analysis of cell division by endoribonuclease-prepared siRNAs. Cell Cycle 4:564-567, 2005

34. Kuwahara T, Koyama A, Koyama S, Yoshina S, Ren CH, Kato $\mathrm{T}$, et al: A systematic RNAi screen reveals involvement of endocytic pathway in neuronal dysfunction in alpha-synuclein transgenic C. elegans. Hum Mol Genet 17:2997-3009, 2008

35. Lamb J, Crawford ED, Peck D, Modell JW, Blat IC, Wrobel MJ, et al: The connectivity map: using gene-expression signatures to connect small molecules, genes, and disease. Science 313:1929-1935, 2006

36. Leal JF, Ferrer I, Blanco-Aparicio C, Hernández-Losa J, Ramón Y Cajal S, Carnero A, et al: S-adenosylhomocysteine hydrolase downregulation contributes to tumorigenesis. Carcinogenesis 29:2089-2095, 2008

37. Lents NH, Baldassare JJ: RNA interference takes flight: a new RNAi screen reveals cell cycle regulators in Drosophila cells. Trends Endocrinol Metab 17:173-174, 2006

38. Loh SHY, Francescut L, Lingor P, Bähr M, Nicotera P: Identification of new kinase clusters required for neurite outgrowth and retraction by a loss-of-function RNA interference screen. Cell Death Differ 15:283-298, 2008

39. Lu PY, Xie FY, Woodle MC: Modulation of angiogenesis with siRNA inhibitors for novel therapeutics. Trends Mol Med 11:104-113, 2005

40. Luo J, Emanuele MJ, Li D, Creighton CJ, Schlabach MR, Westbrook TF, et al: A genome-wide RNAi screen identifies multiple synthetic lethal interactions with the ras oncogene. Cell 137:835-848, 2009

41. Ma C, Mandrekar SJ, Alberts SR, Croghan GA, Jatoi A, Reid $\mathrm{JM}$, et al: A phase I and pharmacologic study of sequences of the proteasome inhibitor, bortezomib (PS-341, Velcade), in combination with paclitaxel and carboplatin in patients with advanced malignancies. Cancer Chemother Pharmacol 59: 207-215, 2007

42. MacKeigan JP, Murphy LO, Blenis J: Sensitized RNAi screen of human kinases and phosphatases identifies new regulators of apoptosis and chemoresistance. Nat Cell Biol 7:591-600, 2005

43. Martinez J, Patkaniowska A, Urlaub H, Lührmann R, Tuschl T: Single-stranded antisense siRNAs guide target RNA cleavage in RNAi. Cell 110:563-574, 2002

44. Mi H, Guo N, Kejariwal A, Thomas PD: PANTHER version 6: protein sequence and function evolution data with expanded representation of biological pathways. Nucleic Acids Res 35 (Database issue):D247-D252, 2007

45. Morgan-Lappe S, Woods KW, Li Q, Anderson MG, Schurdak ME, Luo Y, et al: RNAi-based screening of the human kinome identifies Akt-cooperating kinases: a new approach to designing efficacious multitargeted kinase inhibitors. Oncogene 25:1340-1348, 2006

46. Mori R, Xiong S, Wang Q, Tarabolous C, Shimada H, Panteris $\mathrm{E}$, et al: Gene profiling and pathway analysis of neuroendocrine transdifferentiated prostate cancer cells. Prostate 69:12-23, 2009

47. Mukherji M, Bell R, Supekova L, Wang Y, Orth AP, Batalov $\mathrm{S}$, et al: Genome-wide functional analysis of human cell-cycle regulators. Proc Natl Acad Sci U S A 103:14819-14824, 2006

48. Orth AP, Batalov S, Perrone M, Chanda SK: The promise of genomics to identify novel therapeutic targets. Expert Opin Ther Targets 8:587-596, 2004

49. Oyaizu H, Adachi Y, Okumura T, Okigaki M, Oyaizu N, Ta- 
ketani S, et al: Proteasome inhibitor 1 enhances paclitaxelinduced apoptosis in human lung adenocarcinoma cell line. Oncol Rep 8:825-829, 2001

50. Parsons DW, Jones S, Zhang X, Lin JCH, Leary RJ, Angenendt $P$, et al: An integrated genomic analysis of human glioblastoma multiforme. Science 321:1807-1812, 2008

51. Pollack I: Growth factor signaling pathways and receptor tyrosine kinase inhibitors, in Newton HB (ed): Handbook of Brain Tumor Chemotherapy. Philadelphia: Elsevier, 2006, pp 155-172

52. Ramadan N, Flockhart I, Booker M, Perrimon N, Mathey-Prevot B: Design and implementation of high-throughput RNAi screens in cultured Drosophila cells. Nat Protoc 2:22452264, 2007

53. Raychaudhuri B, Han Y, Lu T, Vogelbaum MA: Aberrant constitutive activation of nuclear factor kappaB in glioblastoma multiforme drives invasive phenotype. J Neurooncol 85:39-47, 2007

54. Rhodes DR, Kalyana-Sundaram S, Mahavisno V, Varambally $\mathrm{R}, \mathrm{Yu}$ J, Briggs BB, et al: Oncomine 3.0: genes, pathways, and networks in a collection of 18,000 cancer gene expression profiles. Neoplasia 9:166-180, 2007

55. Robinson R: RNAi therapeutics: how likely, how soon? PLoS Biology 2(1):e28, 2004

56. Russ AP, Lampel S: The druggable genome: an update. Drug Discov Today 10:1607-1610, 2005

57. Sachse C, Echeverri CJ: Oncology studies using siRNA libraries: the dawn of RNAi-based genomics. Oncogene 23:83848391,2004

58. Sathornsumetee S, Reardon DA: Targeting multiple kinases in glioblastoma multiforme. Expert Opin Investig Drugs 18:277-292, 2009

59. Schultz N, Lopez E, Saleh-Gohari N, Helleday T: Poly(ADPribose) polymerase (PARP-1) has a controlling role in homologous recombination. Nucleic Acids Res 31:4959-4964, 2003

60. Sepp KJ, Hong P, Lizarraga SB, Liu JS, Mejia LA, Walsh CA, et al: Identification of neural outgrowth genes using genomewide RNAi. PLoS Genet 4:e1000111, 2008

61. Short SMC, Mayes C, Woodcock M, Johns H, Joiner MC: Low dose hypersensitivity in the T98G human glioblastoma cell line. Int J Radiat Biol 75:847-855, 1999

62. Stein GH: T98G: an anchorage-independent human tumor cell line that exhibits stationary phase G1 arrest in vitro. J Cell Physiol 99:43-54, 1979

63. Meier S, Gehring C: A guide to the integrated application of on-line data mining tools for the inference of gene functions at the systems level. Biotechnol J 3:1375-1387, 2008

64. Sudheerkumar P, Shiras A, Das G, Jagtap JC, Prasad V, Shastry P: Independent activation of Akt and NF-kappaB pathways and their role in resistance to TNF-alpha mediated cytotoxicity in gliomas. Mol Carcinog 47:126-136, 2008

65. Tai AW, Benita Y, Peng LF, Kim SS, Sakamoto N, Xavier RJ, et al: A functional genomic screen identifies cellular cofactors of hepatitis $\mathrm{C}$ virus replication. Cell Host Microbe 5: 298-307, 2009
66. Tang D, Kehrer J: Carcinogenesis: balance between apoptosis and survival pathways, in Srivastava R (ed): Apoptosis, Cell Signaling, and Human Diseases: Molecular Mechanisms. Totowa NJ: Humana Press, 2007, Vol 1

67. Tang W, Dodge M, Gundapaneni D, Michnoff C, Roth M, Lum L: A genome-wide RNAi screen for Wnt/beta-catenin pathway components identifies unexpected roles for TCF transcription factors in cancer. Proc Natl Acad Sci U S A 105:9697-9702, 2008

68. Thaker NG, Zhang F, McDonald PR, Shun TY, Lewen MD, Pollack IF, et al: Identification of Survival Genes in Human Glioblastoma Cells Using siRNA Screening. Mol Pharmacol [epub ahead of print], 2009

69. Tu Z, Argmann C, Wong KK, Mitnaul LJ, Edwards S, Sach IC, et al: Integrating siRNA and protein-protein interaction data to identify an expanded insulin signaling network. Genome Res 19:1057-1067, 2009

70. Turner NC, Lord CJ, Iorns E, Brough R, Swift S, Elliott R, et al: A synthetic lethal siRNA screen identifying genes mediating sensitivity to a PARP inhibitor. EMBO J 27:1368-1377, 2008

71. Valster A, Tran NL, Nakada M, Berens ME, Chan AY, Symons M: Cell migration and invasion assays. Methods 37:208-215, 2005

72. Weiss GJ, Kingsley C: Pathway targets to explore in the treatment of non-small cell lung cancer. Journal of Thoracic Oncology 3:1342-1352, 2008

73. Weller MRJ, Rieger J, Grimmel C, Van Meir EG, De Tribolet N, Krajewski S, et al: Predicting chemoresistance in human malignant glioma cells: the role of molecular genetic analyses. Int J Cancer 79:640-644, 1998

74. Westbrook TF, Martin ES, Schlabach MR, Leng Y, Liang AC, Feng B, et al: A genetic screen for candidate tumor suppressors identifies REST. Cell 121:837-848, 2005

75. Whitehurst AW, Bodemann BO, Cardenas J, Ferguson D, Girard L, Peyton M, et al: Synthetic lethal screen identification of chemosensitizer loci in cancer cells. Nature 446:815-819, 2007

76. Zhang JH, Chung TD, Oldenburg KR: A simple statistical parameter for use in evaluation and validation of high throughput screening assays. J Biomol Screen 4:67-73, 1999

77. Zhang Z, Jiang G, Yang F, Wang J: Knockdown of mutant Kras expression by adenovirus-mediated siRNA inhibits the in vitro and in vivo growth of lung cancer cells. Cancer Biol Ther 5:1481-1486, 2006

Manuscript submitted September 12, 2009

Accepted October 27, 2009.

Address correspondence to: Nikhil G. Thaker, B.S., 601 Riverside Avenue, Apartment 424, Lyndhurst, New Jersey, 07071. email: thakerng@umdnj.edu. 